\title{
Surfing on a herringbone
}

\author{
Dan Soto, Guillaume Lagubeau, Christophe Clanet, and David Quéré \\ Physique et Mécanique des Milieux Hétérogènes, UMR No. 7636 du CNRS, ESPCI, 75005 Paris, France \\ and LadHyX, UMR No. 7646 du CNRS, École Polytechnique, 91128 Palaiseau Cedex, France
}

(Received 12 January 2016; published 12 May 2016)

\begin{abstract}
Evaporation of a liquid on a hot solid can be exploited to induce both levitation and self-propulsion if the vapor flow under the liquid is made directional. Here we propose to force an anisotropic flow of vapor with a herringbone pattern at a solid surface. Liquids are found to self-propel on such hot materials, drawn by the underlying flow of vapor. The simplicity of the geometry allows us to predict the direction of vapor flow rectification, to quantitatively model the propulsion, and to discuss how the pattern must be designed to optimize both driving force and drop velocity.
\end{abstract}

DOI: 10.1103/PhysRevFluids.1.013902

\section{INTRODUCTION}

Volatile liquids on hot substrates levitate upon their vapor in the so-called Leidenfrost state [1-3]. These hovercrafts persist a long time due to the insulating properties of the underlying cushion and glide with negligible friction [4]. They can be controlled by macrotextures that enhance friction [5], modify impact [6,7], and induce self-propulsion if displayed as a ratchet [8]. Asymmetric hot teeth, known at room temperature to induce anisotropic liquid adhesion $[9,10]$ or motion if vibrated [11], rectify the vapor flow under the drop, which generates propulsion of the hovercraft $[8,12-15]$. The effect is enhanced with boiling $[8,16]$ and it similarly propels sublimating platelets $[12,15,17,18]$. These systems are original heat transporters, since they both absorb heat and get readily evacuated in a predefined direction.

The underlying mechanism explaining propulsion has recently been the subject of much debate. A wide variety of different scenarios has been considered: viscous entrainment by the vapor $[8,12,13]$, liquid interface deformation [8], inertial thrust [17], nucleate boiling [16], or thermal creep [19]. Careful study of the gas flow and the order of magnitude of the force allows considering the viscous entrainment scenario as a reasonable mechanism [12,14,15]. However, quantitative studies in the ratchet geometry have a major limitation: The local deformation of the bottom of the droplet interface due to the texture is complex and even the main direction of the vapor flow, essential information for understanding the propelling mechanism, is not obvious a priori.

Previous studies on Leidenfrost self-propelling objects have limited their scope to the ratchet geometry. However, we can suspect that any texture forcing an anisotropic evacuation of vapor might generate propulsion. Here we propose to test this general idea by geometrically channeling vapor in a herringbone pattern. Hence, drops interact with grooves instead of teeth. This simple geometry permits us to describe explicitly the flow of vapor at the texture scale and thus to settle the debate on the propelling mechanism. We discuss the force and speed generated by such devices and how they can be used to manipulate levitating liquids by accelerating, stopping, and guiding them.

\section{EXPERIMENT}

The experimental platform is a block of brass $(15 \mathrm{~cm}$ long, $6 \mathrm{~cm}$ wide, and $2 \mathrm{~cm}$ thick) machined to create a pattern likely to both channel the vapor flow and make it asymmetric. As the simplest solution to satisfy these specifications, we dig parallelepiped grooves (depth $H=0.2 \mathrm{~mm}$ and width $W=1 \mathrm{~mm}$ ) separated by walls of thickness $\lambda=0.2 \mathrm{~mm}$ and organized with a herringbone pattern (Fig. 1). Tolerance on the grooves is $10 \mu \mathrm{m}$ and the tip angle $2 \alpha$ at the intersection of two channels can be varied by steps of $30^{\circ}$ between $0^{\circ}$ and $180^{\circ}$, two extreme values defining grooves parallel 

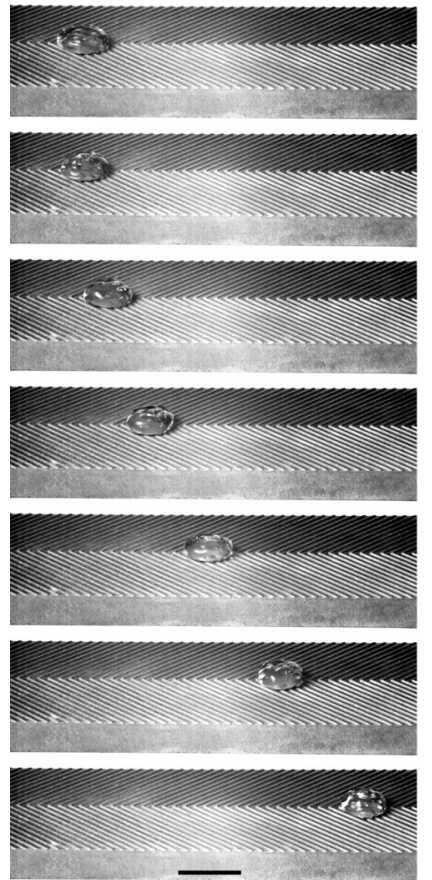

(a)

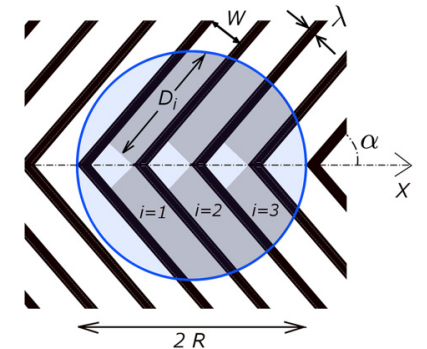

(b)

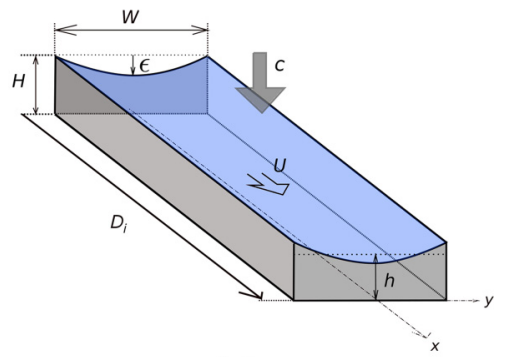

(c)

FIG. 1. Self-propulsion on a herringbone. (a) Placed on the axis of a hot herringbone etched in brass (angle $2 \alpha=90^{\circ}$ and $T=400^{\circ} \mathrm{C}$ ), a drop of acetone (mass $M$ and volume $\Omega=200 \mu \mathrm{L}$ ) self-propels. The drop accelerates (acceleration $a$ ), from which the propelling force $F=M a$ can be deduced. Each crenel has a depth $H=0.2 \mathrm{~mm}$ and a width $W=1 \mathrm{~mm}$. The wall thickness $\lambda$ between channels is $0.2 \mathrm{~mm}$. The bar shows $1 \mathrm{~cm}$ and the interval between images is $0.2 \mathrm{~s}$. (b) Top sketch of the device, with the drop in blue and the definition of the various geometric parameters. (c) Channel of vapor (in gray) bounded by a curved liquid-vapor interface (in blue). The vapor flows at an average velocity $U$, while vapor is injected at a velocity $c$ from the top.

or perpendicular to the herringbone axis. Drops of acetone (mass $M$ and equatorial radius $R$ ) are deposited on this axis after having brought brass to a temperature $T$ of typically $400{ }^{\circ} \mathrm{C}$. As shown in Fig. 1(a), the liquid is observed to exit in the direction opposite to the arrows made by the pattern.

The time interval between pictures in Fig. 1(a) is constant and we can see that the liquid accelerates before reaching a stationary velocity $V \approx 10 \mathrm{~cm} / \mathrm{s}$. In order to extract the propelling force $F$ from the acceleration $a$, we throw drops at a velocity $V_{0} \approx 5 \mathrm{~cm} / \mathrm{s}$ in the direction opposite to propulsion: The liquid slows down, stops, and accelerates backward (see movie 1 in [20]). The corresponding trajectory $X(t)$ is parabolic $\left[X(t)=V_{0} t-a t^{2} / 2\right]$, from which we extract the acceleration $a$ and the propelling force $F=M a$. Drops lose about $1 \mathrm{mg} / \mathrm{s}$ by evaporation, so their mass $M(\approx 100 \mathrm{mg})$ can be taken constant during experiments performed in typically $1 \mathrm{~s}$.

We show in Fig. 2(a) how the propelling force $F$ varies with the liquid radius $R$. The minimum radius corresponds to drops slightly larger than the channel width $W$, a condition for achieving propulsion. Hence radii are all around or above the capillary length $\ell$, which simplifies the liquid geometry: As shown in Figs. 1 and 3, drops are puddles of radius $R$ and height $2 \ell$, the thickness of nonwetting puddles [5]. Acetone at its boiling point $\left(56^{\circ} \mathrm{C}\right)$ has a surface tension $\gamma=19 \mathrm{mN} / \mathrm{m}$ and a density $\rho_{l}=751 \mathrm{~kg} / \mathrm{m}^{3}$, which gives $\ell=1.6 \mathrm{~mm}$.

As observed in Fig. 2(a), the propelling force increases with the drop size. Here $F$ is typically $5 \mu \mathrm{N}$, only a few percent of the liquid weight, yet large enough to generate velocities of a few $\mathrm{cm} / \mathrm{s}$ owing to ultralow adhesion and friction of the system. The force is also a function of the herringbone 


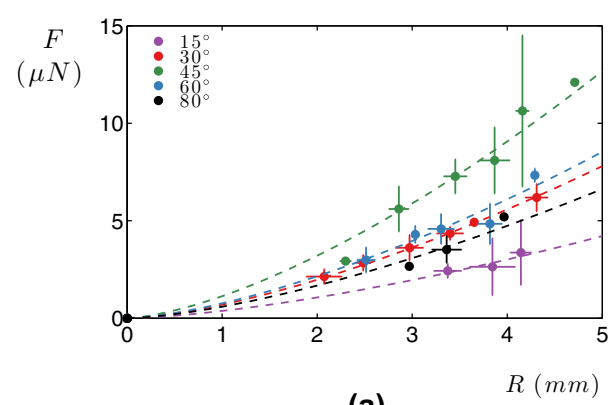

(a)

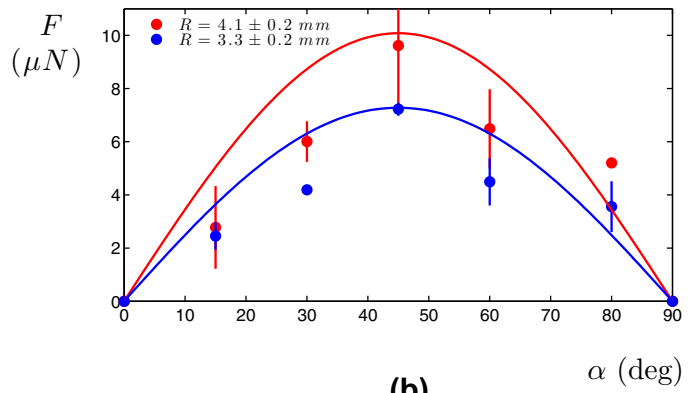

(b)

FIG. 2. Force $F$ propelling acetone drops on hot herringbones. (a) Force $F$ as a function of the drop radius $R$. Each data point is an average of three to five experiments and colors correspond to various pattern angles $2 \alpha$ (with $\alpha=15^{\circ}, 30^{\circ}, 45^{\circ}, 60^{\circ}$, and $80^{\circ}$ ). The channel's width and depth are $W=1 \mathrm{~mm}$ and $H=0.2 \mathrm{~mm}$, respectively, and the wall thickness between channels is $\lambda=0.2 \mathrm{~mm}$. Dotted lines show the behaviors in $R^{3 / 2}$ suggested by Eq. (3). (b) Force $F$ as a function of the pattern angle $\alpha$, for two drop sizes. The fits show Eq. (3) drawn for $R=4.1 \mathrm{~mm}$ (red line) and $R=3.1 \mathrm{~mm}$ (blue line) with a numerical coefficient of 0.8 .

angle $\alpha$ and it seems to be maximum around the median angle $\alpha=45^{\circ}$, as shown in Fig. 2(b) for two drop radii.

\section{COMPLEMENTS}

It is worth completing this information by looking at drops deformations. Macrotextures are known to induce unique dynamical effects, such as drop trapping and splitting [5,6], or enhanced water repellency $[6,7]$. In each case, local deformations of interfaces play a major role in the dynamic outcome of the liquid. In our case, the texture dictates both the vapor flow and the friction of moving drops. The groove pattern makes it possible to describe quantitatively the shape of the interfaces, contrasting with ratchets of more complex geometry. We show in Fig. 3(a) the side view of a drop on hot, deep, straight channels with width $W$ varying from $0.8 \mathrm{~mm}$ at the center to $1.4 \mathrm{~mm}$ at the periphery.

Figure 3(a) shows that a liquid can be either suspended on close enough crenels (crenels 2-6) or impaled when the width $W$ is too large (crenels 1 and 7). The value of the critical width between these two states can be deduced from analyzing Fig. 3(b), where the radius of the curved interface between two walls is denoted by $r$ and the sagging by $\varepsilon$. These two distances are geometrically related to each other by the relationship $2 r \varepsilon=\varepsilon^{2}+W^{2} / 4$. Equilibrium of suspended interfaces is

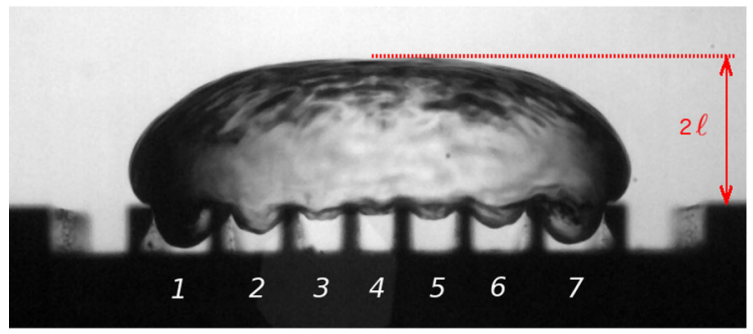

(a)

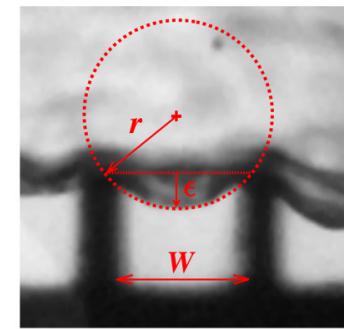

(b)

FIG. 3. Liquid on a crenelated surface. (a) Centimeter-size drop of acetone on a hot substrate $\left(T=400^{\circ} \mathrm{C}\right)$ with straight deep crenels of various widths $W$. Here $W$ is $1.4 \mathrm{~mm}$ for crenels 1 and 7, $1.2 \mathrm{~mm}$ for 2 and $6,1 \mathrm{~mm}$ for 3 and 5, and $0.8 \mathrm{~mm}$ for 4 . The sagging $\varepsilon$ of the liquid increases with $W$ and the transition of impalement occurs for crenels 1 and 7. (b) Zoom in of crenel 5 and definition of the distances relating to the liquid-vapor interface. 




(a)

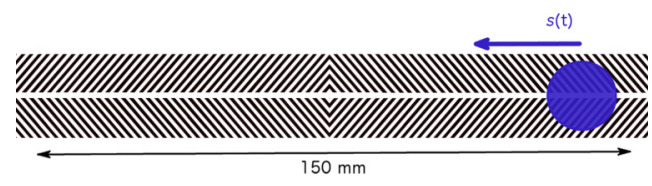

(c)

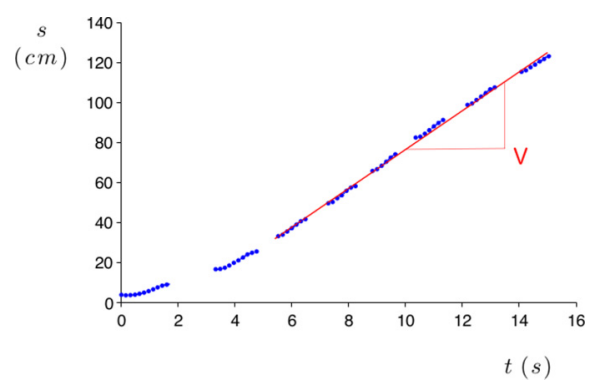

(b)

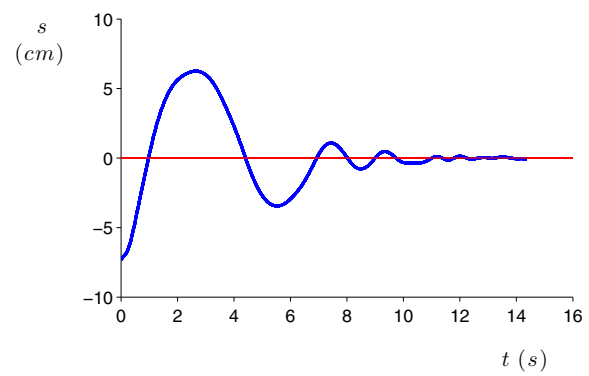

(d)

FIG. 4. Playing with herringbones. (a) Long races are obtained by having two herringbones connected by flat semicircular tracks (see movie 2 in [20]). The curved lines are lateral walls that guide the liquid in the flat region. (b) Drop position along the race, as a function of time for acetone of radius $R=3 \mathrm{~mm}$ (at midrace) and herringbone angle $\alpha=45^{\circ}$. Blue data points recorded along the herringbones show that the terminal velocity $V \approx 10 \mathrm{~cm} / \mathrm{s}$ is reached after approximately one herringbone length. (c) Two herringbones placed head to tail constitute an efficient trap for a drop, as shown in the drop trajectory (d) and in movie 3 in [20].

possible if the Laplace pressure $\gamma / r$ can balance the hydrostatic pressure $\rho_{l} g(2 \ell+\varepsilon)$. Hence we get $\varepsilon \approx W^{2} / 4 \ell$, in the limit of small $\varepsilon(\varepsilon \ll \ell$ and $\varepsilon \ll W)$. Sagging logically increases with the crenel width and the critical $W_{c}$ at which the liquid falls in the texture $(r \approx \varepsilon)$ is obtained for $r=W / 2$. This yields $W_{c}=2(\sqrt{2}-1) \ell$, that is, $2 \mathrm{~mm}$ for water and $1.3 \mathrm{~mm}$ for acetone, whose small surface tension favors impalement. This value fits with what can be seen in Fig. 3(a), where acetone only penetrates in crenels with width $W=1.4 \mathrm{~mm}>W_{c}$.

This discussion has two consequences. First, it justifies how we selected the channel geometry. The width $W$ must be larger than the wall thickness $\lambda=200 \mu \mathrm{m}$ and smaller than $W_{c}$ and we chose $W=1 \mathrm{~mm}$. As seen above, this fixes $\varepsilon=140 \mu \mathrm{m}$ and thus the depth $H$. If we had $H \gg \varepsilon$ [as in Fig. 3(a) where $H=1 \mathrm{~mm}$ ], vapor would not be efficiently confined; conversely, a liquid with small $H$ would levitate far above the textures, making vapor rectification negligible. Hence we took $H=200 \mu \mathrm{m}$, a value slightly larger than $\varepsilon$. Second, it indicates how to keep the liquid across the symmetry axis of the herringbone: If we etch along this axis a deep $(H=800 \mu \mathrm{m})$ straight channel of width $W=1.4 \mathrm{~mm} \geqslant W_{c}$ [Fig. 4(a)], drops will be gravitationally trapped. Such a rail is reminiscent of the ones used in microfluidic to guide liquids [21] and we checked that its presence does not significantly affect the propelling force $F$. Hence, focusing on the local deformation of the liquid not only justifies the choice of the texture dimensions, but also allows us to access and control the confinement height $h$, a key parameter in our problem.

The use of rails allows us to drive drops by long distances, larger than $1 \mathrm{~m}$, as shown with circuits consisting of herringbones in series connected by semicircular tracks bounded by lateral walls [Fig. 4(a)] (see movie 2 in [20]). Drops hardly decelerate in the curved sections and thus readily reach their terminal velocity $V$ (around $10 \mathrm{~cm} / \mathrm{s}$ ). The motion remains stationary [Fig. 4(b)] until the drop size becomes by evaporation comparable to $W_{c}$, which stops the motion. 


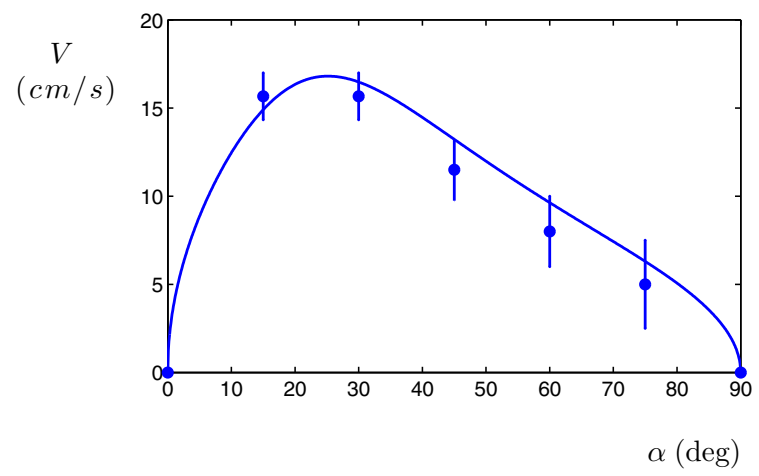

FIG. 5. Drop terminal velocity $V$ as a function of the herringbone (half) angle $\alpha$. Drops are made of acetone, the substrate temperature is $400^{\circ} \mathrm{C}$, and the velocity is measured on circuits consisting of successive herringbones [Fig. 4(a)]. Each point is an average on a distance of about $1 \mathrm{~m}$ and the fit shows Eq. (4) with a numerical coefficient of 0.8 and drawn for $R=3 \mathrm{~mm}$, the drop size at midrace.

Two herringbones can also be displayed with an opposite polarization [Fig. 4(c)] (see also movie 3 in [20]), which makes drops oscillate. Oscillations are damped by friction and the levitating liquid eventually gets immobilized at the center [Fig. 4(d)]: Head-to-tail herringbones constitute efficient traps for Leidenfrost drops.

The device in Fig. 4(a) can be used to determine (and optimize) the terminal velocity $V$, for which propulsion is balanced by friction. As shown in Fig. 5, $V$ decreases by a factor 3 as $\alpha$ increases from $15^{\circ}$ to $75^{\circ}$ for drops of radius $R=3 \pm 1 \mathrm{~mm}$. Unlike $F(\alpha)$ in Fig. 2(b), $V(\alpha)$ is strongly asymmetric, showing a maximum roughly equidistant between $45^{\circ}$, the angle of maximum driving force, and $0^{\circ}$, at which grooves are aligned with the motion, hence minimizing friction.

\section{MODEL}

In order to model this series of observations, we consider a single channel of length $D_{i}$ [Figs. 1(b) and 1(c)] and denote by $U$ and $h(H-\varepsilon<h<H)$ the vapor average velocity and mean thickness. Vapor is injected from the top at a velocity $c$. Assuming $c$ to be independent of the position $x$ along the channel, mass conservation can be written $(h U)_{x+d x}-(h U)_{x}=c d x$. Conduction at temperatures between $200^{\circ} \mathrm{C}$ and $500^{\circ} \mathrm{C}$ dominates heat transfer [2]. For a vapor of density $\rho$ and thermal conductivity $\kappa$, a latent heat of evaporation $\mathcal{L}$, and a difference $\Delta T$ between the substrate temperature $T$ and liquid boiling point, the energy balance per unit time and per unit area can be written $\rho \mathcal{L} c=\kappa \Delta T / h$. Together with mass conservation, this yields the variation of vapor velocity along the channel, taking $x=0$ at the chevron tip:

$$
U=\frac{\kappa \Delta T}{\rho \mathcal{L} h^{2}} x
$$

Vapor in herringbones is channeled towards the grooves exit and the fact that the levitating liquid above is driven in the same direction leads us to interpret the driving force as resulting from viscous drag, as suggested in [8,12-15] and in contradiction with the scenario of inertial thrust proposed in [17]. For each groove, we can integrate along coordinates $x$ and $y$ the viscous stress $\sigma \approx 6 \eta U / h$, where the coefficient 6 corresponds to Poiseuille flow in a gap of thickness $h$. Hence we get a force $3 \eta \kappa \Delta T W D_{i}^{2} / \rho \mathcal{L} h^{3}$ per channel of length $D_{i}$. The total force $F$ is deduced by counting the number $N(\alpha)$ of active channels beneath the liquid, as sketched in Fig. 1(b). For thin walls $(\lambda \ll W)$, we have $N(\alpha) \approx 4 R \sin \alpha / W$. Taking the drop radius $R$ as a typical distance $D_{i}$, we find, after projecting 
along the central axis,

$$
F \approx \frac{6 \eta \kappa \Delta T}{\rho \mathcal{L} h^{3}} R^{3} \sin 2 \alpha
$$

The vapor thickness $h$ is comparable to the channel depth $H$ and we expect it to depend on the drop size $R$, as for a Leidenfrost drop on a flat solid [2]. As shown in the Appendix, this relationship is written $h \approx(b R)^{1 / 2}$, where the distance $b=\left(3 \eta \kappa \Delta T / \rho \mathcal{L} \rho_{l} g \ell\right)^{1 / 2}$ is typically $3 \mu \mathrm{m}$. Hence we get an explicit expression for the driving force, which highlights how it relates to the hydrostatic pressure $2 \rho_{l} g \ell$ exerted by the liquid on the vapor:

$$
F \approx 2 \rho_{l} g \ell R^{3 / 2} b^{1 / 2} \sin 2 \alpha .
$$

We predict $F$ to increase as $R^{3 / 2}$ and to be on the order of $10 \mu \mathrm{N}$ for a drop of $3 \mathrm{~mm}$ (and $\kappa \approx 13 \mathrm{~mW} / \mathrm{m} / \mathrm{K}, \eta \approx 20 \mu \mathrm{Pa} \mathrm{s}, \mathcal{L} \approx 500 \mathrm{~kJ} / \mathrm{kg}$, and $\left.\rho \approx 1.8 \mathrm{~kg} / \mathrm{m}^{3}\right)$, two facts agreeing with the data in Fig. 2(a). Equation (3) also has a maximum for $\alpha=45^{\circ}$, as indeed reported in Fig. 2(b) where the fit is quantitative provided we assume a numerical coefficient of 0.8 multiplying the right-hand side of Eq. (3). The measured force is slightly lower than the expected value from Eq. (3), which might be caused by a small slip at the vapor-liquid interface.

The terminal velocity of liquids can also be discussed. Assuming that friction mainly arises from the soft shocks of the bumps visible in Fig. 3 on the crenellation sides, we expect an inertial resistance $F=\beta \rho_{l} R^{2} V^{2}$, where $\beta$ is a numerical coefficient [5]. Using inclined plates with straight crenels, we checked that friction is quadratic in velocity (see the Appendix). We found $\beta_{\|} \approx 0.021 \pm 0.003$ and $\beta_{\perp} \approx 0.109 \pm 0.015$ for liquids moving parallel and perpendicular to crenels, respectively (logically, we have $\beta_{\|} \ll \beta_{\perp}$ ). Friction on herringbones can be decomposed along these directions ( $F=F_{\perp} \sin \alpha+F_{\|} \cos \alpha$ ), where the liquid velocity is $V \sin \alpha$ and $V \cos \alpha$, respectively. Hence we get a strongly nonlinear expression in $\alpha$ for the friction: $F \approx \rho_{l} R^{2} V^{2}\left(\beta_{\perp} \sin ^{3} \alpha+\beta_{\|} \cos ^{3} \alpha\right)$. Balancing this resistance with propulsion (3) yields the terminal velocity of drops:

$$
V \approx(2 g \ell)^{1 / 2}(b / R)^{1 / 4}\left[\frac{\sin 2 \alpha}{\beta_{\perp} \sin ^{3} \alpha+\beta_{\|} \cos ^{3} \alpha}\right]^{1 / 2} .
$$

Drawn with a solid line in Fig. 5 for $R=3 \mathrm{~mm}$, with the same coefficient of 0.8 as in the fit of Fig. 2(b), Eq. (4) is found to nicely adjust the data, with, in particular, an optimum at small acute angles. Maximizing Eq. (4) with respect to $\alpha$ shows that the optimum angle is only a function of the ratio $\beta_{\|} / \beta_{\perp}$. At small angle, $V(\alpha)$ varies as $2 \alpha /\left(\beta_{\perp} \alpha^{3}+\beta_{\|}\right)$, whose maximum is $\left(\beta_{\|} / 2 \beta_{\perp}\right)^{1 / 3}$, that is, $\alpha \approx 25^{\circ}$ (the value given by numerical integration within $2 \%$ ), in good agreement with experiments.

\section{CONCLUSION}

The ability of herringbone patterns to move Leidenfrost drops can be seen as a geometrical proof of a general scenario relating vapor-propelled systems and asymmetric patterns [8,12-15]. This simple geometry allows us to settle the debate on the propelling mechanism of Leidenfrost drops on ratchets and confirms the scenario of viscous drag by vapor. Contrasting with ratchets, the local deformation of interfaces induced by textures can be modeled, which was shown to be crucial for controlling the direction and speed of vapor. Hence we could characterize quantitatively and optimize these propelling devices, a difficult task in the more complex geometry of ratchets. Maximizing the force is useful if it is desired to oppose an existing force (such as gravity); maximizing the speed permits one to enhance the motion and to evacuate quickly the liquid. The corresponding optimal angles are not the same, but they both underline the role of geometry. Smaller temperatures could also be tested, in particular after coating the solid with hydrophobic microtextures, which preserves the Leidenfrost state down to the boiling point of the liquid [22]. Further, since any pattern polarizing the vapor flow should generate propulsion, many other designs should be imagined and tested along these lines to create efficient and reliable self-propelling devices. 


\section{ACKNOWLEDGMENTS}

We thank Tobias Baier, Guillaume Dupeux, Mary-Laure Finen, Steffen Hardt, Hélène de Maleprade, and Charlotte Rasser for many valuable discussions.

\section{APPENDIX}

We complete our paper with additional information related to the friction force on textured solids and to the propelling force on herringbone patterns.

\section{Measurements of the friction coefficients}

In order to access the friction coefficients on textured materials, we machined parallel crenels in a brass block of $200 \times 200 \mathrm{~mm}^{2}$, with the same geometrical characteristics as for herringbones (depth $H$ of $0.2 \mathrm{~mm}$, width $W$ of $1 \mathrm{~mm}$, and wall thickness $\lambda$ of $0.2 \mathrm{~mm}$ ). This plate is heated to a temperature $T=400{ }^{\circ} \mathrm{C}$ and tilted by an angle $\theta$ to the horizontal, so the levitating liquid is subjected to gravity. Acetone drops (of fixed volume $\Omega \approx 200 \mu \mathrm{L}$ and density $\rho_{l}$ ) accelerate and reach their terminal velocity after a few $\mathrm{cm}$, when the horizontal projection of the gravity $\rho_{l} \Omega g \sin \theta$ is balanced by friction. The plate is first displayed with grooves perpendicular to the trajectory (Fig. 6).

The experiment is made for tilt angle $\theta$ between $1^{\circ}$ and $12^{\circ}$, hence changing the drop velocity and consequently the friction force. By recording the motion, we can access the terminal velocity and plot in Fig. 7(a) the (measured) friction as a function of the (measured) terminal velocity. The solid line represents the best parabolic fit on a log-log scale. If we consider an inertial friction $F=\beta \rho_{l} R^{2} V^{2}$, as discussed by Dupeux et al. [5], we deduce the friction coefficient for grooves perpendicular to the trajectory and find $\beta_{\perp} \approx 0.109 \pm 0.015$.

We can similarly access the friction force as a function of the terminal velocity for grooves parallel to the motion [Fig. 7(b)]. Again, the friction law is well described by a law quadratic in velocity, the signature of inertial friction. The corresponding friction coefficient $\beta_{\|}$deduced from the experiments is $\beta_{\|} \approx 0.021 \pm 0.003$, a value significantly smaller than $\beta_{\perp}$. Friction is logically minimized when the motion is parallel to the grooves, which explains why the maximum velocity of self-propelling drops on herringbones is observed at small herringbone angles.

\section{Vapor thickness in channels}

As shown above, using conservation of mass (1) and thermal balance (2) yields a horizontal vapor speed $U=\frac{\kappa \Delta T}{\rho \mathcal{L} h^{2}} x$ [Eq. (3)]. Integrating the Poiseuille equation $\left(12 \eta U / h^{2}=d P / d x\right)$ in a parallelepiped section gives the pressure beneath the drop, using the boundary condition $P(R)=P_{0}$ :

$$
P(x)-P_{0}=\frac{6 \eta \kappa \Delta T}{\rho \mathcal{L} h^{4}}\left(R^{2}-x^{2}\right) .
$$

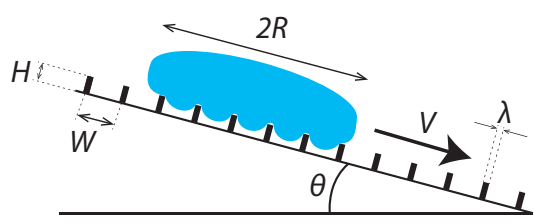

FIG. 6. Drop of acetone with volume $\Omega \approx 200 \mu \mathrm{L}$ and density $\rho_{l}$ descending a crenelated material inclined to the horizontal by an angle $\theta$ of a few degrees. The gravitational force along the motion is $\rho_{l} \Omega g \sin \theta$ and the corresponding terminal velocity is typically $10 \mathrm{~cm} / \mathrm{s}$, comparable to the velocity reached on herringbones. 


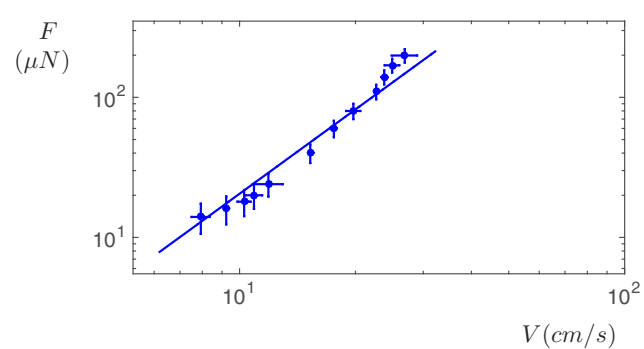

(a)

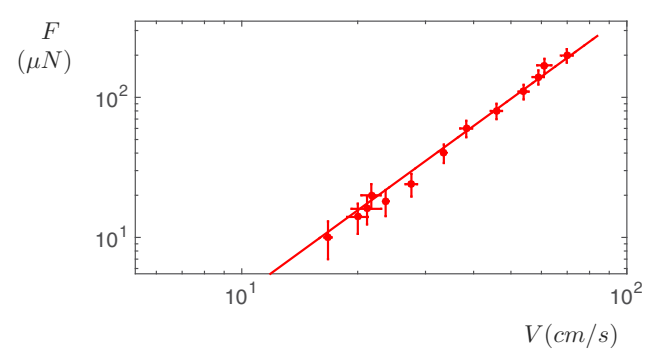

(b)

FIG. 7. Friction as a function of the terminal velocity for grooves (a) perpendicular to the trajectory or (b) parallel to the trajectory. The perpendicular friction is an order of magnitude higher than the parallel one. Both behaviors are quadratic in velocity (inertial friction).

The pressure in the channel at the center of the drop (or at the tip of any chevron) has to balance the hydrostatic pressure $\rho_{l} g 2 \ell$ due to the puddles's weight, leading to $h=\sqrt{b R}$, where the distance $b=\sqrt{\frac{3 \eta \kappa \Delta T}{\rho \mathcal{L} \rho_{l} g \ell}}$ is found to have the same structure as for a drop evaporating on a flat substrate.

\section{Exact calculation of viscous entrainment force}

By symmetry, projections of viscous forces in the plane perpendicular to the axis of the drops motion compensate and they add up in the plane of symmetry. Hence the total entrainment force is

$$
F=2 \cos \alpha \iint_{S} \frac{6 \eta U}{h} d x d y,
$$

where the surface of integration $S$ is half the circle below the drop. To get an analytical solution, we work in the reference frame $\left(\vec{e}_{x}, \vec{e}_{y}\right)$ defined by the direction of the grooves and sketched in Fig. 8 [and different from the reference frame $\left(\vec{e}_{X}, \vec{e}_{Y}\right)$ associated with the plane of symmetry]. Instead of calculating the force generated by the Poiseuille flow in each channel and then adding them up, we directly integrate the stress over the whole surface. Using the expression of the horizontal speed $U=\frac{\kappa \Delta T}{\rho \mathcal{L} h^{2}} x$ in a single channel (3) and denoting $\frac{12 \eta \kappa \Delta T}{\rho \mathcal{L} h^{3}}$ by $A$, the total entrainment force can be rewritten as $F=A \cos \alpha \iint_{S} x d x d y$. We decompose the total integration area $S$ in two regions:

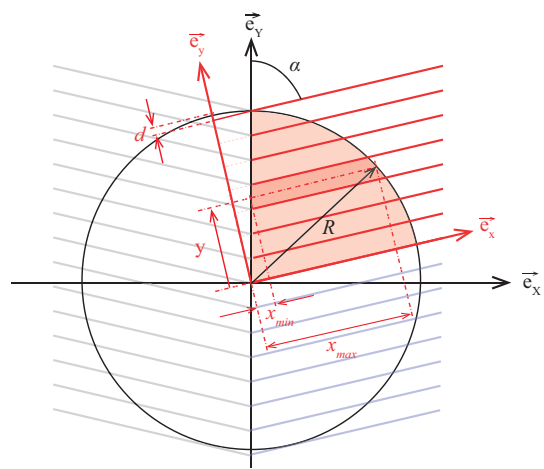

(a)

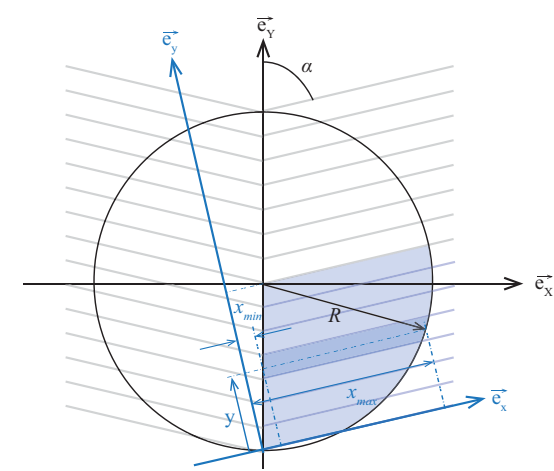

(b)

FIG. 8. Sketch of the surface over which viscous forces are calculated. Here $x_{\min }$ and $x_{\max }$ denote the integral limits in the $\vec{e}_{x}$ direction for a given $y$ position. In the $\vec{e}_{y}$ direction, $y$ is integrated from 0 to $R \sin \alpha$. (a) Upper part, shaded red, corresponds to area $S 1$. (b) Lower part, shaded blue, corresponds to area $S 2$. 
(i) an upper one $S 1$ [in red in Fig. 8(a)], which leads to the integral $\int_{0}^{R \sin \alpha} \int_{y / \tan \alpha}^{\sqrt{R^{2}-y^{2}}} x d x d y$, and (ii) a lower one $S 2$ [in blue in Fig. 8(b)] associated with $\int_{0}^{R \sin \alpha} \int_{y / \tan \alpha}^{\sqrt{R^{2}-(R \sin \alpha-y)^{2}}} x d x d y$. After calculation, we get the following analytical expression for the total entraining force:

$$
F=\frac{6 \eta \kappa \Delta T}{\rho \mathcal{L} h^{3}} R^{3} f(\alpha)
$$

with $f(\alpha)=\cos \alpha\left\{\sin ^{3} \alpha+\frac{1}{8}[6 \cos \alpha(2 \alpha+\sin 2 \alpha)+11 \sin \alpha+3 \sin 3 \alpha]\right\}$ an exact analytical solution very similar to Eq. (4).

[1] J. G. Leidenfrost, De aquae communis nonnullis qualitatibus tractatus (Duisburg, 1756).

[2] B. S. Gottfried, C. J. Lee, and K. J. Bell, Leidenfrost phenomenon-Film boiling of liquid droplets on a flat plate, Int. J. Heat Mass Transfer 9, 1167 (1966).

[3] J. C. Burton, A. L. Sharpe, R. C. A. van der Veen, A. Franco, and S. R. Nagel, Geometry of the Vapor Layer Under a Leidenfrost Drop, Phys. Rev. Lett. 109, 074301 (2012).

[4] I. U. Vakarelski, J. O. Marston, D. Y. C. Chan, and S. T. Thoroddsen, Drag Reduction by Leidenfrost Vapor Layers, Phys. Rev. Lett. 106, 214501 (2011).

[5] G. Dupeux, M. Le Merrer, C. Clanet, and D. Quéré, Trapping Leidenfrost Drops with Crenelations, Phys. Rev. Lett. 107, 114503 (2011).

[6] J. C. Bird, R. Dhiman, H. Kwon, and K. K. Varanasi, Reducing contact time of a bouncing drop, Nature (London) 503, 385 (2013).

[7] Y. Liu, L. Moevius, X. Xu, T. Quian, J. Yeomans, and Z. Wang, Pancake bouncing on superhydrophobic surfaces, Nat. Phys. 10, 515 (2014).

[8] H. Linke, B. J. Aleman, L. D. Melling, M. J. Taormina, M. J. Francis, C. C. Dow-Hygelund, V. Narayanan, R. P. Taylor, and A. Stout, Self-Propelled Leidenfrost Droplets, Phys. Rev. Lett. 96, 154502 (2006).

[9] N. A. Malvadkar, M. J. Hancock, K. Sekeroglu, W. J. Dressick, and M. C. Demirel, An engineered anisotropic nanofilm with unidirectional wetting properties, Nat. Mater. 9, 1023 (2010).

[10] M. J. Hancock, K. Sekeroglu, and M. C. Demirel, Bioinspired directional surfaces for adhesion, wetting, and transport, Adv. Funct. Mater. 22, 2223 (2012).

[11] T. A. Duncombe, E. Y. Erdem, A. Shastry, R. Baskaran, and K. F. Böhringer, Controlling liquid drops with texture ratchets, Adv. Mater. 24, 1545 (2012).

[12] G. Dupeux, M. Le Merrer, G. Lagubeau, C. Clanet, S. Hardt, and D. Quéré, Viscous mechanism for Leidenfrost propulsion on a ratchet, Europhys. Lett. 96, 58001 (2011).

[13] T. R. Cousins, R. E. Goldstein, J. W. Jaworski, and A. I. Pesci, A ratchet trap for Leidenfrost drops, J. Fluid Mech. 696, 215 (2012).

[14] A. G. Marín, D. Arnaldo del Cerro, G. R. B. E. Römer, B. Pathiraj, A. Huis in 't Veld, and D. Lohse, Capillary droplets on Leidenfrost micro-ratchets, Phys. Fluids 24, 122001 (2012).

[15] G. G. Wells, R. McHale, G. Ledesma-Aguilar, and K. A Sefiane, Sublimation heat engine, Nat. Commun. 6, 6390 (2015).

[16] A. Grounds, R. Still, and K Takashina, Enhanced droplet control by transition boiling, Sci. Rep. 2, 720 (2012).

[17] G. Lagubeau, M. Le Merrer, C. Clanet, and D. Quéré, Leidenfrost on a ratchet, Nat. Phys. 7, 395 (2011).

[18] A. Hashmi, Y. Xu, B. Coder, P. A. Osborne, J. Spafford, G. E. Michael, G. Yu, and J. Xu, Leidenfrost levitation: Beyond droplets, Sci. Rep. 2, 797 (2012).

[19] A Würger, Leidenfrost Gas Ratchets Driven by Thermal Creep, Phys. Rev. Lett. 107, 164502 (2011). 
[20] See Supplemental Material at http://link.aps.org/supplemental/10.1103/PhysRevFluids.1.013902 for movies.

[21] P. Abbyad, R. Dangla, A. Alexandrou, and C. N. Baroud, Rails and anchors: Guiding and trapping droplet microreactors in two dimensions, Lab Chip 11, 813 (2011).

[22] I. U. Vakarelski, N. A. Patankar, J. O. Marston, D. Y. C. Chan, and S. T. Thoroddsen, Stabilization of Leidenfrost vapour layer by textured superhydrophobic surfaces, Nature (London) 489, 274 (2012). 